\title{
Modeling technique for vault-like structure generation through topological manipulation
}

XXIV International Conference

of the Iberoamerican Society

of Digital Graphics

Medellin | Colombia

\author{
Marcelo Álvarez \\ Universidad Técnica Federico Santa María | Chile | marcelo.alvarez.13@sansano.usm.cl \\ Marcelo Bernal \\ Perkins\&Will | USA | marcelo.bernal@perkinswill.com \\ Carlos Castro \\ Universidad Técnica Federico Santa María | Chile | carlos.castrog@usm.cl
}

\begin{abstract}
This study is based on the development of a modeling technique for vault-like structure generation through topological manipulation. Currently, topology-driven form-finding has been implemented in tensile structures, but no further studies have been conducted for compression-only structures. The focus of this study is to approach the problem of highly determined vault shapes by their input topology. The technique operates at the topological level between vertices and edges to create an input 2D topology map. The particle-spring system uses such a map to simulate the resulting 3D mesh geometry. For testing purposes, we explore three generative approaches. The results show the effectiveness of the technique to manipulate the topological relationships that controls the generation of the funicular structures.
\end{abstract}

Keywords: Form-finding; Funicular; Particle-spring system; Design space; Topology.

\section{INTRODUCTION}

Funicular shapes are those made by a catenary chain determined by a fixed amount of weights (Addis, 2014). Catenary chains are enabled to design vault-like structures by Robert Hooke's Law of Inversion. Since the term funicular means only tension or only compression this principle can be directly applied to generate structures (Kilian \& Ochsendorf, 2005). Under ideal conditions, forces through a vault-like structure must be axial, with minimal bending. The capacity of manipulating these structures in real-time during the design process enables designers to explore solutions from different configurations. This condition provides an enhanced exploratory nature of the form-finding method.

This study focuses on determining the correlation between the topological manipulation of an initial condition and the funicular vault shape resulting from Particle-spring System Simulation (PSS). Funicular forms are extremely restricted since its geometry is determined by gravity. Form-finding is the method for generating emergent forms from input data. The recent implementation of form-finding techniques is based on the computational simulation of physical models, mainly considering geometrical properties.

Recent studies of topology-driven form-finding by Suzuki \& Knippers (2017a, 2017b, 2017c) focus on tensile structures as they can be actively manipulated by modifying the structural behavior of the initial condition. However, there are no major studies related to funicular only compression vault-like structures. This kind of structure behaves passively under gravity load with no additional external forces. This means the initial condition before a simulation is what defines the possible design space. This study introduces a modeling technique to rationally manipulate the $2 \mathrm{D}$ input topology to induce the generation of tridimensional funicular structures.

\subsection{PARTICLE-SPRING SYSTEM}

Particle-spring System is a form-finding simulation technique that transforms an initial geometry under force load. This system is based on lumped masses (particles) and linear elastic elements (springs) that interconnect particles in space. Kilian and Ochsendorf proposed the utilization of this technique for finding structural forms defined only by axial forces. Multiple applications started from 2005 onwards after the mentioned authors developed the software called CADenary. This software enables the user to modify forms, while continually reaching a state of equilibrium.

Several authors implemented design applications such as: structural network within a three-dimension boundary (Kanellos, 2007), 'Form-responding' variable depth technique (Clifford, 2012), post-formed wooden pavilion (Pone et al., 2013), set of funicular shapes for exploring new design forms (Bhooshan et al., 2014) and anisotropic gridshell structure embedding digital fabrication (Naboni, 2016). Regardless of the field of computation, structure, architecture, or construction), all of them require an initial condition to trigger the Particle-spring system simulation that defines the resulting shape.

\subsection{TOPOLOGY-DRIVEN FORM FINDING}

Geometric processes generate variations through dynamic manipulation of metric parameters without affecting the initial topologic conditions. Instead, Suzuki \& Knippers state that topological processes enable differentiation through interactive adjustments in topological models. This study focuses on the restriction of the initial geometry for vault-like shape generation. Therefore, the concept of topology-driven form-finding is the basis of this study. At 
present, Suzuki \& Knippers implemented the topologydriven form-finding concept in Bending-active tensile hybrid structures (BATH). The authors developed the software called ElasticSpace, and the case study for the AAVS Madrid Pavilion.

Other researchers approached topology manipulation to expand the space design implementing the use of digital tools. Deleuran et al. (2016), using the Kangaroo2 goals method studied the differentiation of forms based on a dynamic topological model. Additionally, Ahlquist et al. (2015) developed a computational environment called springFORM. This environment introduces the concept of 'metatopology': a global topology configuration carrying local topological properties. The specified approaches focus on tensile structures actively allowing the manipulation of form. There are no further studies for vaultlike structures because the generated forms are determined by the initial topology.

To address this constraint, this methodology focuses on generating initial conditions to expand the early-stage design space. Since the vault-like structures are restricted to the initial condition configuration, this technique detaches the topological structure from the threedimensional geometry generation. The methodology proposes the following structure: the regular polygon input generation, the first set of topological manipulation based on the initial polygon, and the second set of topological operations based on vertices and edges. Once the application of all the topological operations occurs, the vertex configuration constructs a triangulated mesh. With a user-defined anchor point selection, a Particle-spring system simulation runs to generate a vault emergent shape.

\section{METHODOLOGY}

This study develops a generative technique based on primitive topological objects. The generative modeling technique does not represent a possible solution. On the contrary, it represents the design space understood as 'explorable and exploitable potential' (Najle, 2004). The primitive topological objects are the vertex and edge. The configuration of these objects defines the initial condition geometry. Adding edges inside the geometry boundary generate discontinuity and subdivision. Adding vertices directly increases vertex count and implicit valency properties. The vertex set defines a point cloud to construct a mesh geometry using Delaunay triangulation. Also, the user can configure the anchor point set. Each step saves the topological information, allowing the algorithm to keep the links between the addition or the deletion of operations. The design space allows the study of the correlation between the initial condition and the Particle-spring system simulation result. Figure 1 illustrates the flow diagram of this study methodology, where function processes are represented with their expected partial results.

\subsection{INPUT GEOMETRY GENERATION: REGULAR N- GON}

The minimal input for this technique to perform is a regular $\mathrm{n}$-gon polygon. The generation of the geometry is based on the equal subdivision of a circumference. If this input generation is simplified, it only needs a unique point/vertex and setting a radius. The minimum valid number of polygon sides is three, and the maximum number defined for this study is eight.

\section{Function Process}
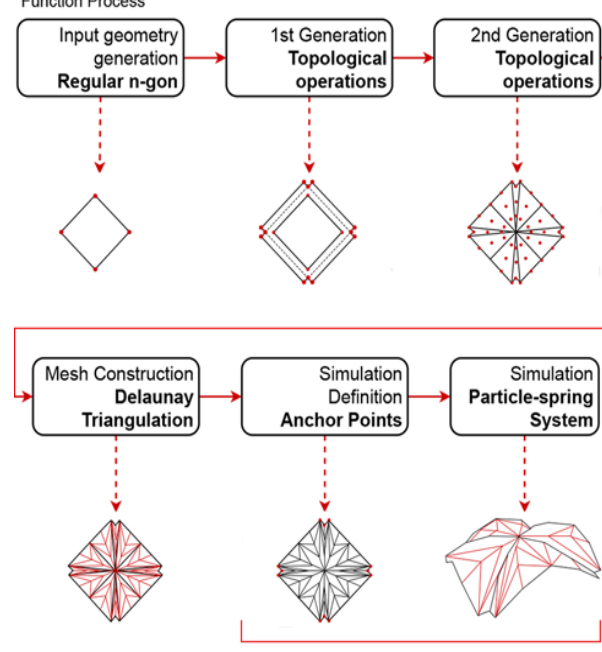

Figure 1: Global process of geometry generation.

\subsection{FIRST GENERATION OF TOPOLOGICAL OPERATIONS}

This set of operations generates the first layer of differentiation between this process outputs and the first polygon. This set of operations consists of geometric manipulation by moving and scaling the polygon. For the topological description of this operation, boundary regions are operated as a Boolean union and intersection, producing the first-generation initial condition (Figure 2). This geometry only includes a boundary as edges and vertices on boundary discontinuities.

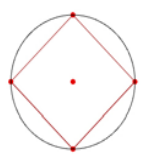

0. Circumference Subdivision

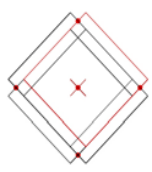

1. Move and copy Initial polygon

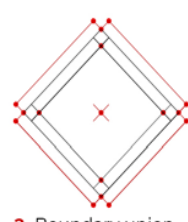

2. Boundary union Vertex definition
Figure 2: Primitive polygon geometry generation and transformation.

\subsection{SECOND GENERATION OF TOPOLOGICAL OPERATIONS}

Vertices and edges define the initial condition configuration. The edge is an implicit property that describes the system connectivity and the boundary curve condition. For this study, the primitive initial condition is the regular $n$-gon polygon. This operation evaluates an " $n$ " number of vertices on a circumference. The topological properties of this initial condition are the polygon edge quantity and vertex amount and area centroid. The boundary condition determines three possible operation spaces: the area inside the boundary, the area outside the boundary, and the explicit edges of the boundary (Figure 3). 

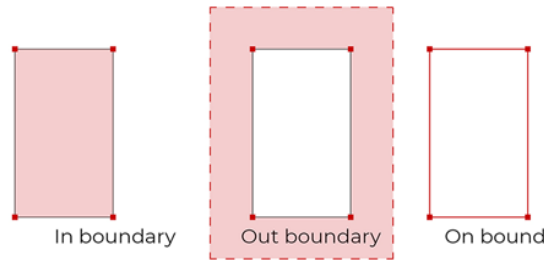

On boundary

Figure 3: Boundary condition defined by initial edge configuration.

Edges enable vertices to be operated on, as they control the unidimensional position based on the initial geometry regardless of any geometrical property. For the cases mentioned previously, edges can be added as inner edges, outer edges, or using the initial edge with no edge addition. The addition of edges requires two vertices; therefore, the area centroid is one of the vertices this edge definition needs. This method generates a new edge by connecting it to the midpoint of each starting edge in the geometry (Figure 4).

Figure 5 illustrates the set of topological operations described. Operations for edges occur before vertex operations to control and limit the two-dimensional space of manipulation. This control that vertices can only be operated on edges, principally by adding vertices because of the growing nature of this modeling technique.
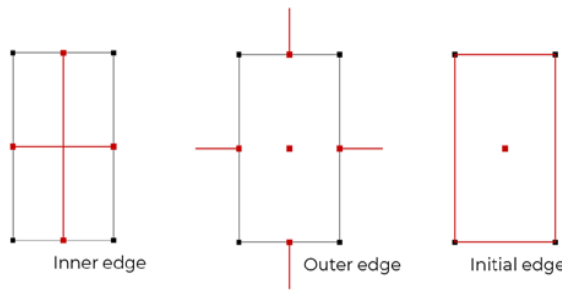

Figure 4: Edge operations on initial boundary condition.
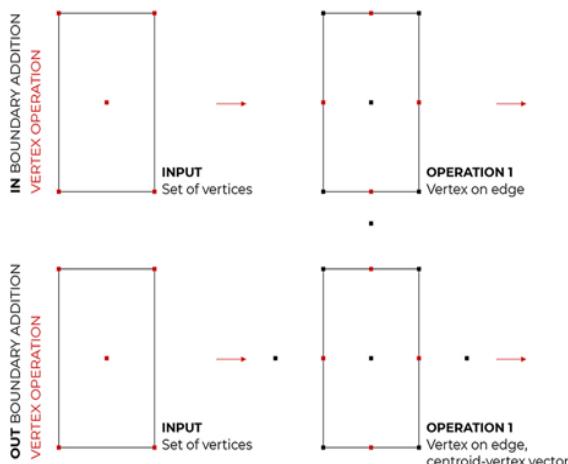

Set of vertices
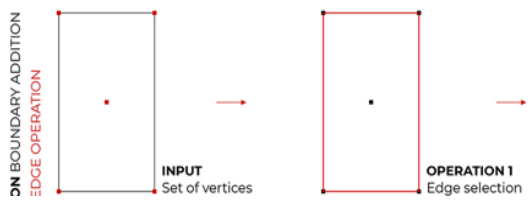
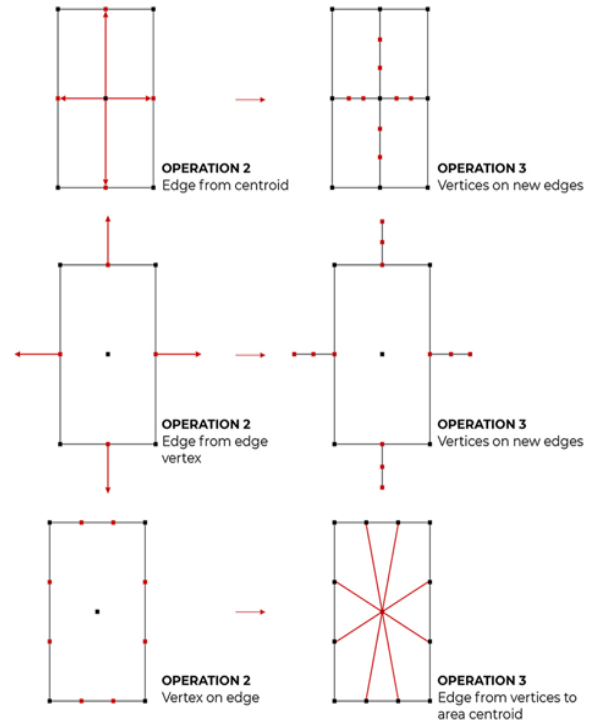

Figure 5: Operation set for topological manipulation

\subsection{DELAUNAY TRIANGULATION FOR MESH CONSTRUCTION}

The given set of vertices obtained from the topological operations determines the mesh construction process. Delaunay triangulation takes the point cloud in a plane and internally makes groups of 3 vertices each, defining circumferences. No other vertex exists inside that circumference. The advantages of using Delaunay triangulation are the no vertex specific order requirement, and the maximization of the minimum angle for all the triangle faces of the mesh, avoiding narrow angles (Figure $6)$.

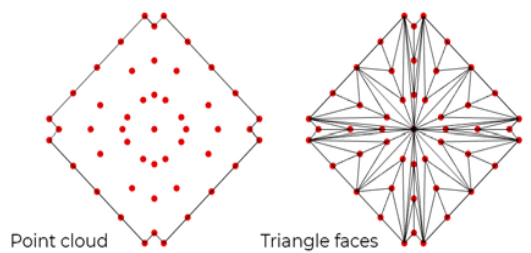

Figure 6: Delaunay mesh configuration for a given set of vertices.

\subsection{MESH ANCHOR POINT DEFINITION AND SIMULATION}

Anchor points are the most defining entity for emergent shapes outside of the simulation parameter control. Vault shapes can be highly differentiated by activating or deactivating vertices. For this study, the area centroid used through all the topological manipulation processes is preserved. Besides the vertices can be activated or deactivated, the area centroid, edge vertices, and interior vertices determine anchor points.

The Particle-spring system simulation additionally requires the unary force vector simulating the gravity force and springs properties definition. From the initial condition geometry, edges are linear elastic elements; or springs. 
Springs are linear elastic elements and must have a rest length factor. The rest length is the final extension the spring tries to reach. Also, the user must provide a stiffness coefficient value depending on the general scale of the model. Stiffness coefficient is the ratio of the force applied on a linear element and the displacement from the equilibrium state. This methodology defines an initial stiffness value of 25 , but since it is variable the user can modify this value. The stiffness coefficient does not interfere with the topological operation process. (Figure 7).

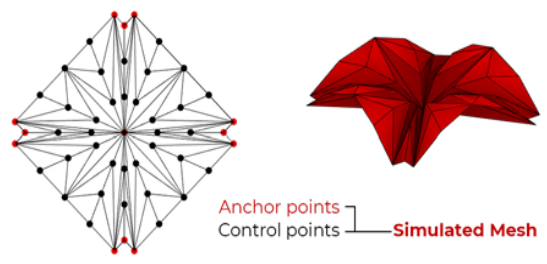

Figure 7: Anchor point definition for PSS mesh simulation.

\subsection{MODELING TECHNIQUE IMPLEMENTATION}

The Particle-spring system simulation in Kangaroo Physics by Daniel Piker (2013) within the Grasshopper-Rhinoceros environment supports this technique. Additionally, iterations for design space exploration is supported by Colibri by Thornton Tomasetti. The geometric manipulation defines metric parameters within the PSS Kangaroo environment: stiffness, spring length factor, and unary vector force as gravity. The parameters specified can influence the geometric qualitative properties of the resulting forms.

Since the technique focuses on manipulating the initial condition only, the addition/subtraction of vertices within the boundary condition domain defines the topological properties. The topological properties are the vertex valency, system connectivity, and continuity of shapes. Generating a two-dimensional and a three-dimensional set of geometry tests this technique's capacity to generate emergent shapes. Also, the topology generated is a modular unit for the propagation and composition of more complex two-dimensional plans.

\section{RESULTS}

The results of this technique implementation are the twodimensional topological configuration geometry and threedimensional vault-like geometry, simulated with the Particle-spring System technique. The two-dimensional geometry results include the topological configuration and the triangulated mesh. While the topological configuration contains vertices and edges, the mesh represents the system connectivity. On the other hand, the threedimensional vault-like geometry contains the particle and spring system actioned by applying a simulated gravity force.

\subsection{D TOPOLOGY RESULTS}

The 2D geometries are the result of applying topological operations of addition under a primitive state of a regular ngon polygon. These individual operations, in order, are the following: number of polygon sides, polygon growing composition, vertex-edge subdivision, interior, and exterior vertex addition. The specified parameters (Table 1) evaluate the generated topological configurations within the range of described parameters.
Table 1: Topological configuration variables with specified range of evaluation. The range with decimal values set intervals of 0.25 (quarter).

\begin{tabular}{lll}
\hline Variable & Description & Range \\
\hline polygon & Sides number & {$[3-8]$} \\
copyScale & Scale factor & {$[0.25-1.00]$} \\
copyMove & Vector factor & {$[0.50-1.25]$} \\
ptEdge & On edge add & {$[0-3]$} \\
ptlnt & In bound add & {$[0-3]$} \\
ptExt & Out bound add & {$[0-3]$} \\
\hline
\end{tabular}

A general rule of sampling defines these domain ranges. Excluding the polygon variable, the user can modify all other parameters using a quarter of the domain size as steps. Table 1 illustrates decimal numbers of the parameter copyScale that can be $0.25,0.50,0.75,1.00$. The same rule applies to the copyMove variable. For the ptEdge, ptInt and ptExt variables, as integers, possible values for evaluation are $0,1,2$ and 3 .

The size of this selectively constrained space design is 6144 solutions. This experiment selects a total of 128 geometry samples of two-dimensional and threedimensional geometries. For the topology configuration circles in red denote vertices, while black lines represent edges. The Delaunay triangulated mesh stores the information of each boundary, defined by the previous topological operations. Figure 8 illustrates sample results with respective parameter configuration.
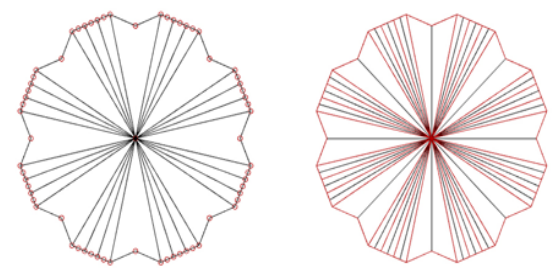

INDEX 27

polygon

copyScale copyMove ptEdge ptInt
ptExt 3
0.75 0.75
0.50 faceCount area 3 ptExt

0
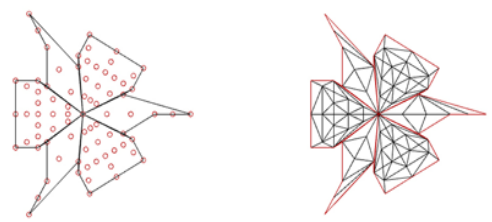

INDEX 31

$\begin{array}{llll}\text { polygon } & 3 & \text { faceCount } & 126 \\ \text { copyScale } & 0.75 & \text { area } & 1623.79 \mathrm{~cm}^{2} \\ \text { copyMove } & 0.50 & & \\ \text { ptEdge } & 3 & & \\ \text { ptInt } & 3 & & \\ \text { ptExt } & 2 & & \end{array}$




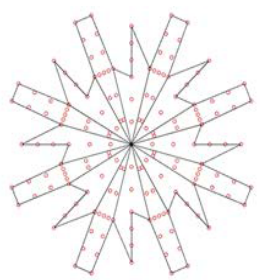

INDEX 124

$\begin{array}{ll}\text { polygon } & \mathbf{8} \\ \text { copyScale } & \mathbf{1 . 0 0} \\ \text { copyMove } & \mathbf{0 . 5 0} \\ \text { ptEdge } & \mathbf{3} \\ \text { ptInt } & \mathbf{2} \\ \text { ptExt } & \mathbf{3}\end{array}$

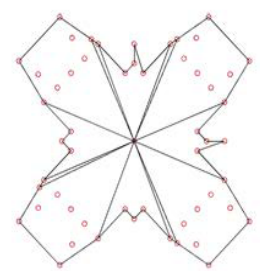

INDEX 55

$\begin{array}{llll}\text { polygon } & 4 & \text { faceCount } & \mathbf{7 4} \\ \text { copyScale } & 1.00 & \text { area } & 4111.33 \mathrm{~cm}^{2} \\ \text { copyMove } & 0.75 & & \\ \text { ptEdge } & \mathbf{2} & & \\ \text { ptInt } & 1 & & \\ \text { ptExt } & \mathbf{2} & & \end{array}$

Figure 8: Topology configuration results with a triangulated mesh topology.

\subsection{D TOPOLOGY RESULTS}

Each set of topology configurations also have a tridimensional vault-like structure geometry. Regarding the space design size of the two-dimensional catalog, the size of the three-dimensional space is 110592. The design space increases because of including the anchor point manipulation process. The simulation of the twodimensional topologies combines the following parameters: a gravity load of $9.8 \mathrm{~m} / \mathrm{s} 2$, stiffness of 25 for the current scale of the model, and a spring rest length factor of 1 . This section refers only to the topological module and the vault emergent shape. Shown below are the respective $3 \mathrm{D}$ models of the results sampled above (Figure 9).

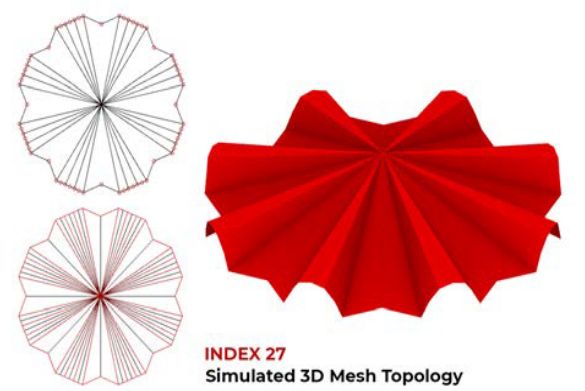

Simulated 3D Mesh Topology

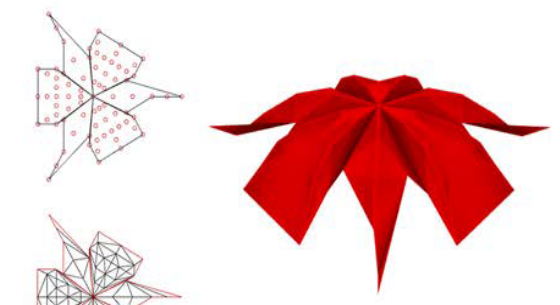

INDEX 31

Simulated 3D Mesh Topology
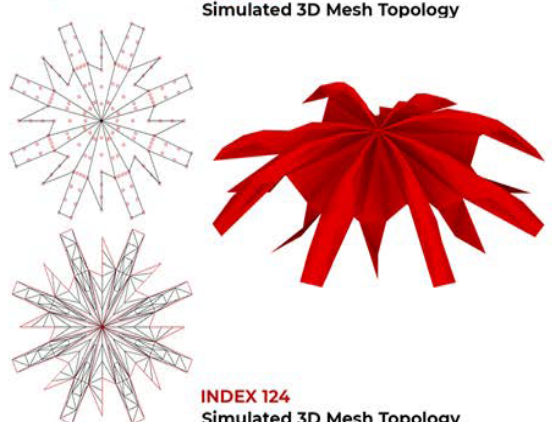

Simulated 3D Mesh Topology
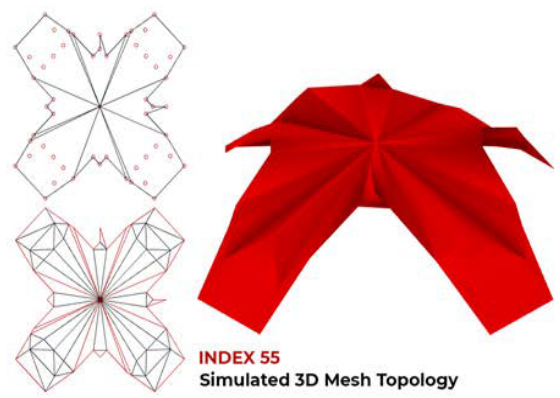

INDEX 55

Simulated 3D Mesh Topology

Figure 9: 3D vault-like shapes. Geometry generated from the 2D configuration.

\subsection{MODULAR COMPOSITION METHODS}

The overall process is a continuous increase of layers to develop the complexity of the entire system. Therefore, this study proposes a modular composition method to use as geometry input to implement the modeling technique. The topological operation set is global for a given polygonal geometry composition to generate a new initial condition and mesh configuration with an emergent shape from the Particle-spring system simulation. The first composition method is a semi-regular polygonal tiling with a concentric arrangement. The second composition method uses a Voronoi cell configuration inside a regular boundary condition. The third composition method is a reproduction exercise of Gaudi's Sagrada Familia cathedral using the modular initial condition results.

\subsubsection{SEMI-REGULAR POLYGONAL TILING COMPOSITION METHOD}

A semi-regular tiling is a tessellation made of two or more regular polygons. Although not all polygonal combinations can create a valid semi-regular composition, this method performs a Boolean intersection to split and make a non- 
overlapping composition. Figure 10 shows four samples with a main central polygon and a radial arrangement of secondary polygons. This method uses the resulting set of boundary condition and vertices to construct a mesh by merging the boundaries into a single region or preserving the polygonal module information (Figure 11). As a result, two possible configurations of $3 \mathrm{D}$ vault-like forms emerge from the 2D mesh topology. Figure 12 shows a single merged mesh, while Figure 13 illustrates a multiple modular mesh composition.
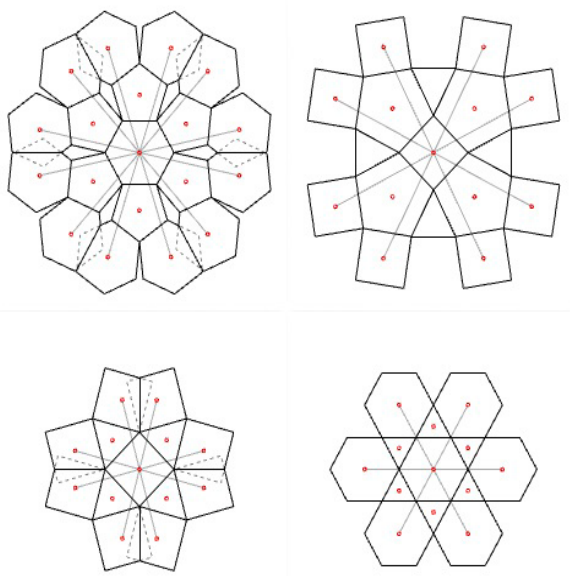

Figure 10: Semi-regular polygonal tiling with vectors and centroids.
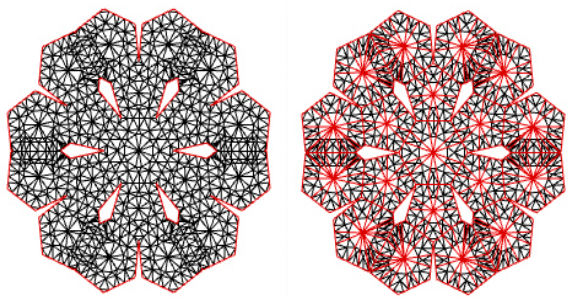

Figure 11: Left: Merged mesh. Right: Modular mesh.

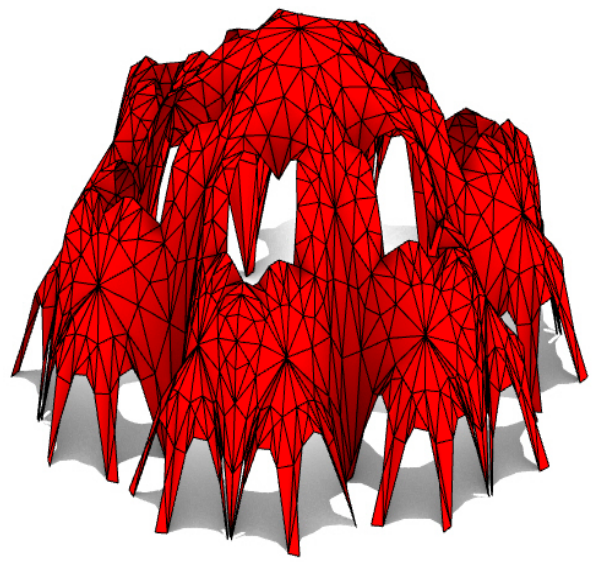

Figure 12: 3D merged vault-like mesh result.

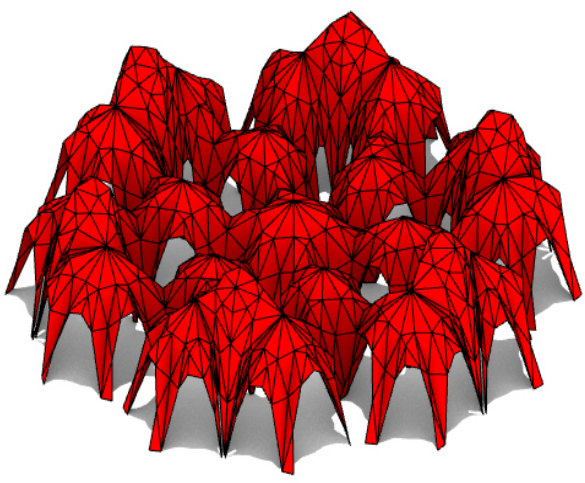

Figure 13: 3D modular vault-like mesh result.

\subsubsection{VORONOI CELL CONFIGURATION COMPOSITION METHOD}

A Voronoi diagram is the dual-graph of a Delaunay triangulation. This diagram allows a non-regular polygonal composition from a non-uniform vertex distribution on a $2 \mathrm{D}$ plane. A boundary condition enables the domain of the Voronoi cells, although it is possible to cull the outer cells to obtain an irregular boundary condition composition (Figure 14). Like the previous study, this method allows a merged mesh construction and modular mesh construction to differentiate the 3D results (Figure 15). Figure 16 shows the $3 \mathrm{D}$ resulting shape of the modular Voronoi cell mesh, and Figure 17 shows a single merged mesh.
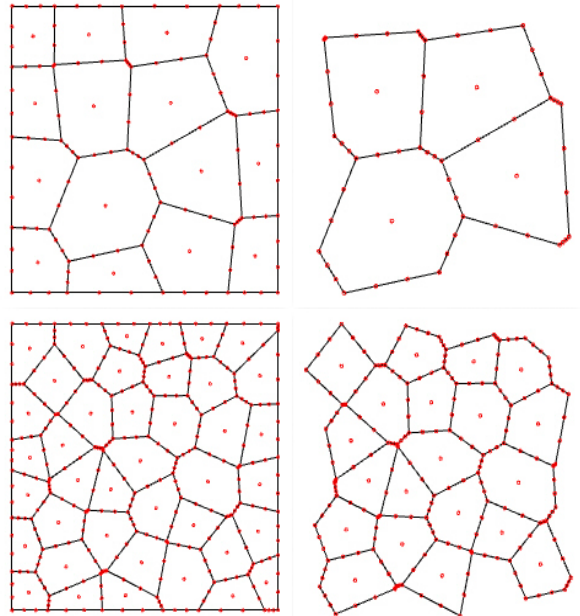

Figure 14: Voronoi cell composition with different boundaries.
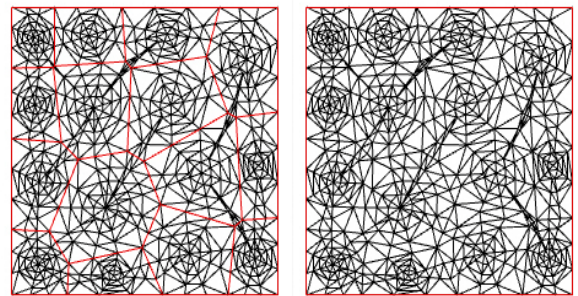

Figure 15: Left: Modular mesh. Right: Merged mesh. 


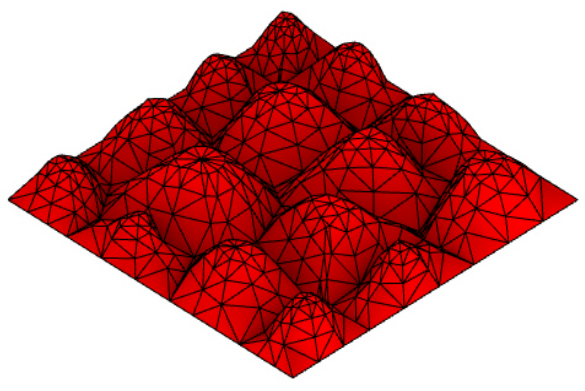

Figure 16: 3D modular vault-like mesh result.

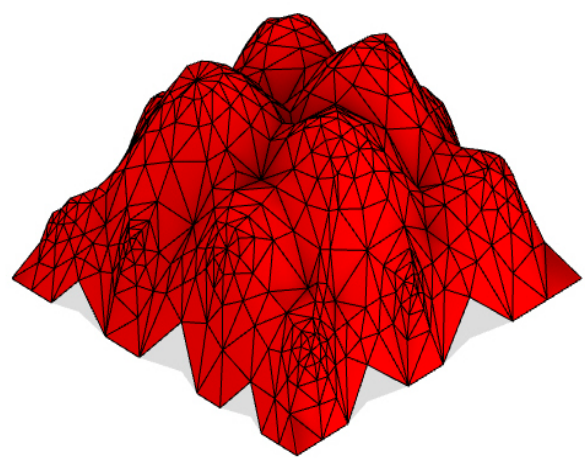

Figure 17: 3D merged vault-like mesh result.

\subsubsection{GAUDI'S SAGRADA FAMILIA CATHEDRAL REPRODUCTION}

Since the modeling technique is capable of generating $2 \mathrm{D}$ topological configurations, the floor plan of the Gaudi's cathedral allows the placement of initial condition meshes (Figure 18). This reproduction study uses vertex and boundary condition information from the floor plan and places the dome vertices at a fixed height. Figure 19 illustrates the $3 \mathrm{D}$ result where an iterative calibration of weight factors for gravity load simulates the vault-like geometry. As this study approaches architecture directly, Figure 20 shows a section diagram of the 3D result.

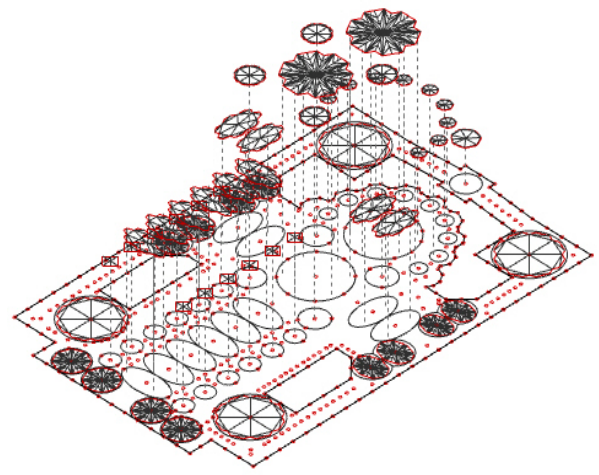

Figure 18: Sagrada Familia floor plan with fixed vertices at plane and mesh domes at fixed heights.

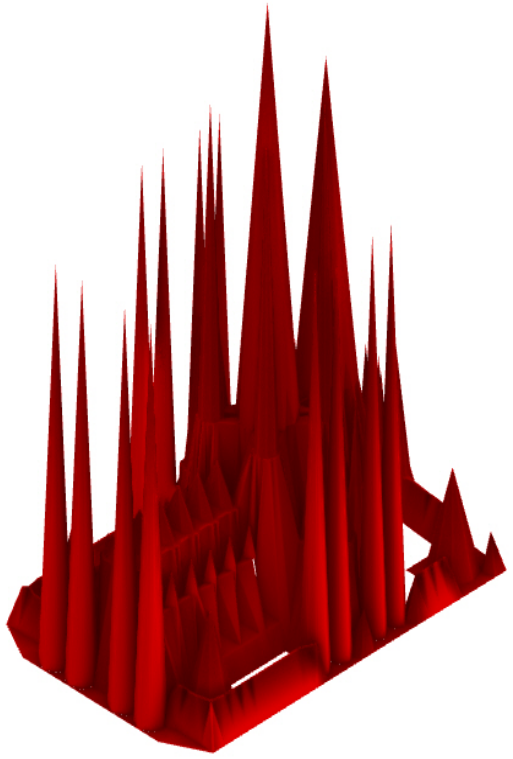

Figure 19: 3D mesh result of the Sagrada Familia reproduction.

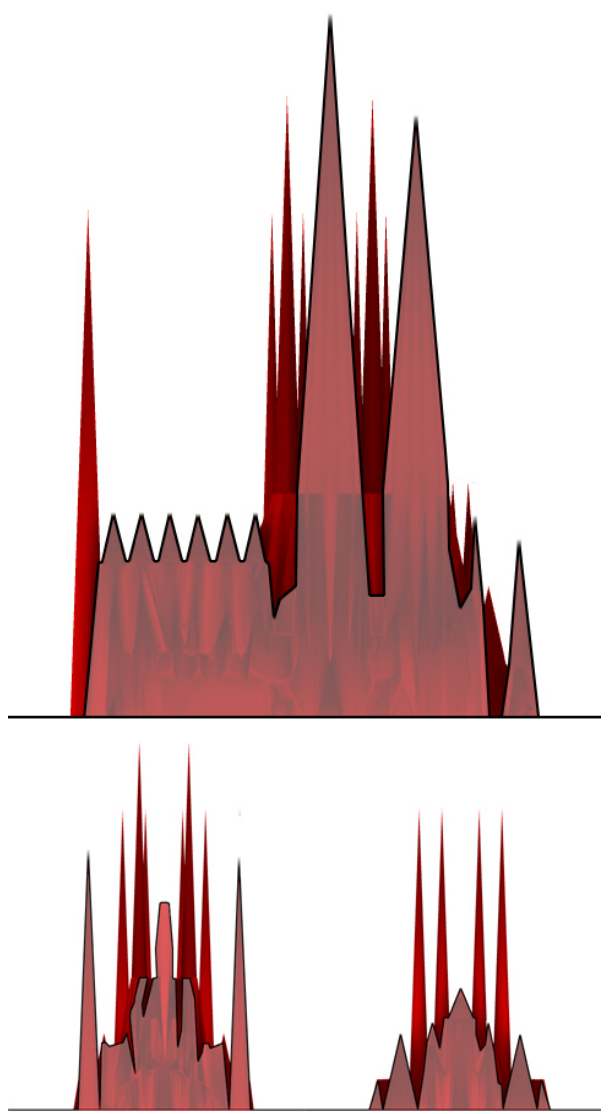

Figure 20: Section diagram of the Sagrada Familia reproduction. 


\section{DISCUSSION}

The development of this technique focuses on expanding the early-stage design space. This technique would allow designers to generate topological configurations as initial conditions separately of the three-dimensional geometry generation. Currently, early form-finding processes focus on the three-dimensional geometry, constraining the initial condition to a user-defined state. The main contribution of this technique is the formal language based on a topological operation that grants a whole catalog of vaultlike structures. Also, detaching the topological configuration from the vault-like shape generation allows the control of the emergent form by manipulating a set of edges and vertices. This study tests the potential of result differentiation for space design extension.

The composition methods explored in this study expands the design space by testing more complex geometry. The semi-regular polygonal tiling can generate topologies by propagating a primitive polygon. For each composition result, vertices can be operated on to set up the mesh construction technique. This would allow the generation of emergent forms from the same initial condition. On the other hand, the Voronoi cell method allows a non-regular 2D topological configuration from a given set of vertices. This study tests the modeling technique under an irregular initial condition set. Finally, the Gaudi's Sagrada Familia cathedral reproduction merges all the resources this modeling technique employs to approach a more complex architecture. Once provided the required topological information, this method would allow the generation of $3 \mathrm{D}$ vault-like complex structures using the modular initial condition from the early stage process.

This study focuses only on the geometrical side of the formfinding for funicular structures design space. One of the main features of using a funicular form-finding technique is the inherent structural optimization approach. Further development for this technique is associating the structural behavior properties to the topological configuration. The main goal is to discover more optimal topological configurations and recognizing which parameter setting can generate more reliable structures. Optimizing this technique to generate only those defined as structurally efficient geometries would allow designers to manipulate topology in later design stages.

\section{REFERENCES}

Addis, B. (2014). Physical modelling and form finding. In S. Bhooshan, D. Veenendaal, \& P. Block, Shell Structures for Architecture, form finding and optimization (pp. 33-43). New York, NY: Routledge.

Adriaenssens, S., Block, P., Veenendaal, D., \& Williams, C. (2014). Shell Structures for Architecture: Form Finding and Optimization. Routledge

Ahlquist, S., Erb, D., \& Menges, A. (2015). Evolutionary structural and spatial adaptation of topologically differentiated tensile systems in architectural design. In Artificial Intelligence for Engineering Design, Analysis and Manufacturing (pp. 393415). Cambridge University Press.

Bertin, T. B. (2011). Evaluating the Use of Particle-Spring Systems in the Conceptual Design of Grid Shell Structures. Worcester Polytechnic Institute.

Bhooshan, S., Veenendaal, D., \& Block, P. (2014). Particle-spring systems: Design of a cantilevering concrete shell. In S.
Adriaenssens, P. Block, D. Veenendaal, \& C. Williams, Shell Structures for Architecture: Form Finding and Optimization (pp. 103-113).

Block, P., Lachauer, L., \& Rippmann, M. (2014). Thrust Network Analysis: Design of a cut-stone Masonry Vault. In S. Adriaenssens, P. Block, D. Veenendaal, \& C. Williams, Shell Structures for Architecture: Form Finding and Optimization (pp. 71-87).

Clifford, B. (2012). Thick Funicular: Particle-Spring Systems for Variable-Depth Form-Responding Compression-Only Structures. Princeton University / The Ohio State University.

Cook, R. (1974). Concepts and Applications of Finite Element Analysis.

Deleuran, A. H., Pauly, M., Tamke, M., Tinning, I. F., \& Thomsen, M. R. (2016). Exploratory Topology Modelling of Form-Active Hybrid Structures. In Procedia Engineering (pp. 71-80).

Goldsmith, N. S. (2014). Shape Finding or Form Finding? In Shells, Membranes and Spatial Structures: Footprints. Brasilia, Brazil.

Kanellos, A. (2007). Topological Self-Organisation: Using a particle-spring system simulation to generate structural spacefilling lattices. London.

Kilian, A., \& Ochsendorf, J. (2005). Particle-Spring Systems for Structural Form Finding. Journal of the International Association for Shell and Spatial Structures: IASS.

Laiserin, J. (2008). Digital Environments for Early Design: FormMaking versus Form-Finding. First International Conference on Critical Digital: What Matter (s)? (pp. 235-242).

Lewis, W. J. (2003). Tension structures: Form and Behavior. London, Westminster, London: ICE Publishing.

Naboni, R. (2016). Form-finding to fabrication of super-thin anisotropic gridshell. Milan, Italy: SIGraDi 2016, XX Congress of the Iberoamerican Society of Digital Graphics.

Pone, S., Colabella, S., Parenti, B., D., L., \& Fiore, A. (2013). Construction and form-finding of a post-formed timber gridshell.

Suzuki, S., \& Knippers, J. (2017). Topology-driven Form-finding: Implementation of an Evolving Network Model for Extending Design Spaces in Dynamic Relaxation. Stuttgart, Germany: Institute of Building Structures and Structural Design, University of Stuttgart.

Suzuki, S., \& Knipperss, J. (2017). Topology-driven Form-finding: Implementation of an Evolving Network Model for Extending Design Spaces in Dynamic Relaxation. In Protocols, Flows and Glitches, Proceedings of the 22nd International Conference of the Association for Computer-Aided Architectural Design Research in Asia (pp. 489-499). Hong Kong: CAADRIA

Suzuki, S., \& Knippers, J. (2017). The Design Implications of Form-Finding with Dynamic Topologies. Stuttgart, Germany: Institute of Building Structures and Structural Design, University of Stuttgart.

Najle, C. (2004). Machinic manifesto. Quaderns d'arquitectura i urbanisme. Volume 244 Q 4.0 (pp. 127-136)

Piker, D. (2013). Kangaroo: Form Finding with Computational Physics. Architectural Design, 83(2), (pp. 136-137).

Thornton Tomasetti Second Colibri Release, CORE studio. 